PROCEEDINGS OF THE

AMERICAN MATHEMATICAL SOCIETY

Volume 129, Number 8, Pages 2467-2471

S 0002-9939(00)05820-2

Article electronically published on December 7, 2000

\title{
ISOSPECTRALITY AND 3-MANIFOLD GROUPS
}

\author{
DANIEL RUBERMAN \\ (Communicated by Ronald A. Fintushel)
}

\begin{abstract}
The Chern-Simons and $\eta$-invariants are closely related invariants of a Riemannian 3-manifold. A difference in their behavior under taking a finite covering space is exploited to give an obstruction to a group being the fundamental group of a closed 3-dimensional manifold.
\end{abstract}

\section{INTRODUCTION}

In this note, we explain how a well-known construction of isospectral manifolds leads to an obstruction to a group being the fundamental group of a closed 3dimensional manifold. The problem of determining, for a given group $G$, whether there is a closed 3-manifold $M$ with $\pi_{1}(M) \cong G$ is readily seen to be undecidable; let us write $G \in \mathcal{G}^{3}$ if there is such a 3-manifold. A standard conjecture (related to Thurston's geometrization program) states that the only possible finite groups in $\mathcal{G}^{3}$ are those which act freely and linearly on $S^{3}$; cf. [Tho86. Algebraic ideas (which apply to the analogous problem in high-dimensional topology [DM85]) come close to proving this conjecture, although there are groups which are not ruled out by surgery theory but which do not act linearly. The new invariant we construct seems to be related to the classical surgery obstructions, but we do not know the precise relationship.

\section{An inVARIANT OF A GRoup AND AN OBSTRUCTION}

Let us say that Riemannian manifolds $\left(M_{1}, g_{1}\right)$ and $\left(M_{2}, g_{2}\right)$ are isospectral on forms if the Laplace operators on the ( $L^{2}$-completions of $) \Omega^{k}\left(M_{i}\right)$ have the same spectrum (counting multiplicities) for all $k$. There is a beautiful construction of pairs of isospectral manifolds, due to Sunada [Sun85], which starts with the following group-theoretical concept.

Definition 2.1. Let $G$ be a finite group. Two subgroups $H$ and $K$ are said to be almost-conjugate if any of the following conditions holds:

1. Every element in $H$ is conjugate to an element of $K$.

2. For each conjugacy class $C$ in $G$, the intersections $C \cap H$ and $C \cap K$ have the same cardinality.

3. The permutation representation of $G$ on $G / H$ (acting by left multiplication) is conjugate to the permutation representation of $G$ on $G / K$.

Received by the editors March 16, 1998 and, in revised form, December 6, 1999.

2000 Mathematics Subject Classification. Primary 57M10, 58J28.

The author was partially supported by NSF Grant 4-50645.

(C)2000 American Mathematical Society 
It is worth noting that the last version could equally be applied to subgroups $K$ and $H$ of finite index in a possibly infinite group $G$.

Suppose that $M$ is a Riemannian manifold, that $H, K$ are subgroups of $G$, and that $\varphi: \pi_{1}(M) \rightarrow G$ is a surjection. Let $M_{H}$ denote the the covering space of $M$ corresponding to the subgroups $\varphi^{-1}(H)$, with a similar definition for $M_{K}$. Sunada showed Sun85, Bér92 that if $H$ and $K$ are almost-conjugate, then $M_{H}$ and $M_{K}$ are isospectral on forms. There are many examples of almost-conjugate subgroups; we will recall one in the last section.

The other ingredients in our obstruction are two related invariants of a Riemannian 3-manifold: the $\eta$-invariant APS75a, and the Chern-Simons invariant CS74]. The $\eta$-invariant is a real-valued Riemannian invariant, which is defined using the spectrum of the Laplacian on 1-forms of a Riemannian 3-manifold. In particular, two 3-manifolds which are isospectral on forms must have the same $\eta$-invariant. The Chern-Simons invariant takes its values in $\mathbf{R} / \mathbf{Z}$; the main property we will need is that the Chern-Simons invariant is multiplicative under finite covers. The $\eta$ invariant determines the Chern-Simons invariant up to an ambiguity of $1 / 2(\bmod \mathbf{Z})$ via the equation [APS75b]

$$
3 \eta(M) \equiv 2 \operatorname{CS}(M) \quad(\bmod \mathbf{Z}) .
$$

Atiyah-Patodi-Singer identify the ambiguity as follows:

Definition 2.2. For any finitely-generated group $\pi$, let $S(\pi) \in\{0,1\}$ denote the number (modulo 2) of two-torsion summands in the abelianization $H_{1}(\pi)$ of $\pi$. For a space $X$, let $S(X)=S\left(\pi_{1}(X)\right)=S\left(H_{1}(X)\right)$.

An elementary application of the universal coefficient theorems shows that

$$
S(\pi)=\operatorname{dim}_{\mathbf{Z}_{2}} H_{1}\left(\pi ; \mathbf{Z}_{2}\right)-\operatorname{dim}_{\mathbf{Q}} H_{1}(\pi ; \mathbf{Q}) .
$$

The precise relationship between Chern-Simons and $\eta$-invariants is then

$$
\frac{3}{2} \eta(M)-\mathrm{CS}(M) \equiv \frac{1}{2} S(M) \quad(\bmod \mathbf{Z}) .
$$

With all these preliminaries out of the way, we can now define an invariant of a group $\pi$.

Definition 2.3. Let $\pi$ be a finitely generated group, and let $\varphi: \pi \rightarrow G$ be a surjection, where $G$ is a finite group with almost-conjugate subgroups $H$ and $K$. Define $\Delta \mathrm{S}(\pi ; \varphi) \in \mathbf{Z}_{2}$ to be

$$
S\left(\varphi^{-1}(H)\right)-S\left(\varphi^{-1}(K)\right) .
$$

If $\Delta \mathrm{S}(\pi ; \varphi)=0$ for all possible $\varphi$, then we will say that $\pi$ satisfies the CS condition.

Theorem 2.4. Suppose that $\pi=\pi_{1}\left(M^{3}\right)$ where $M$ is a closed orientable 3-manifold. Then $\pi$ satisfies the CS condition.

Proof. Given a triple $(G, H, K)$ and a surjection $\varphi: \pi_{1}(M) \rightarrow G$, construct the manifolds $M_{H}$ and $M_{K}$. By definition, the fundamental group of $M_{H}\left(\right.$ resp. $\left.M_{K}\right)$ is $\varphi^{-1}(H)\left(\operatorname{resp} . \varphi^{-1}(K)\right)$, so

$$
\Delta \mathrm{S}(\pi, \varphi)=S\left(M_{H}\right)-S\left(M_{K}\right) .
$$

Since $M_{K}$ and $M_{H}$ are isospectral, they have the same $\eta$-invariant. On the other hand, $M_{K}$ and $M_{H}$ are both finite covering spaces (of the same degree) of $M$, so they have the same CS invariants. It follows from equation (2.2) that $S\left(M_{H}\right)=S\left(M_{K}\right)$, and hence that $\Delta \mathrm{S}(\pi, \varphi)=0$. 
Theorem 2.4 can be interpreted as saying that a finite group $G$, having almost conjugate subgroups $H$ and $K$ with $S(H) \neq S(K)$, cannot act freely on a homotopy sphere. Since the CS condition is homological in nature, it is reasonable to expect that such a statement should hold with weaker hypotheses.

Theorem 2.5. Let $G$ be a finite group, with almost conjugate subgroups $H$ and $K$. If $G$ acts freely on a $\mathbf{Z}_{2}$ homology sphere $M^{3}$, then $S(H)=S(K)$.

Proof. Let $M_{H}=M / H$ and $M_{K}=M / K$ as above. The argument for Theorem 2.4 applies to show that $S\left(M_{H}\right)=S\left(M_{K}\right)$, so we need to see that $S(K)=S\left(M_{K}\right)$, with the analogous statement holding for $H$. According to the remark after the definition of $S$, it suffices to prove that

$$
H_{1}\left(M_{K} ; \mathbf{Q}\right) \cong H_{1}(K ; \mathbf{Q})(=\{0\}) \text { and } H_{1}\left(K ; \mathbf{Z}_{2}\right) \cong H_{1}\left(M_{K} ; \mathbf{Z}_{2}\right) .
$$

The first follows by an elementary transfer argument, and the second follows directly from the spectral sequences for the (regular) covering $M \rightarrow M_{K}$, with $\mathbf{Z}_{2}$ coefficients.

\section{An example}

To find groups which are excluded from membership in $\mathcal{G}^{3}$ by the CS condition, one can simply look among the known examples of almost-conjugate subgroups of finite groups until one finds one for which $S(H) \neq S(K)$.

Example 3.1. One example of this phenomenon was pointed out to me by R. Guralnick. Quoting from a letter of several years ago,

Let $\mathrm{G}=M_{23}$, the Mathieu group of degree 23 . There are two subgroups $H=2^{4} \cdot A_{7}$ (that is there is a normal subgroup of order $2^{4}$ and the quotient is $A_{7}$ ) and $K=L_{3}(4) .2$ which induce the same character (this is evident from the Atlas of finite groups for example). $H$ is perfect so $S(H)=0$, while $K / K^{\prime}=\mathbf{Z} / 2$ so $S(K)=1$.

From this example, one concludes that $M_{23}$ is not the fundamental group of any 3 -manifold, although this is can also be shown by cohomological means.

Example 3.2. The simplest example I know is based on the following observation. Any group of order $n$ acts on itself by left multiplication, and hence imbeds as a subgroup of the symmetric group $S_{n}$. Let $H$ and $K$ be groups of order $n$, which have the same number of elements of order $k$, for any $k \mid n$. Then $H$ and $K$ are almost-conjugate subgroups of $S_{n}$. To apply this, let $H=\mathbf{Z}_{4} \oplus \mathbf{Z}_{2} \oplus \mathbf{Z}_{2}$, which has $S(H)=1$. In the listing of 2-groups of small order [HS64] can be found a group denoted $16 \Gamma_{2} c_{1}$, with the same number of elements of any order as does $H$. (See page 39 of [HS64] for this information, and page 16 for a presentation of the group. With this presentation, I verified the necessary algebraic facts using the computer program GAP $\left[\mathrm{S}^{+} 93\right.$.) Letting $K=16 \Gamma_{2} c_{1}$ which has abelianization $\mathbf{Z}_{4} \oplus \mathbf{Z}_{2}$, we deduce that the symmetric group $S_{16}$ does not satisfy condition CS.

From this example, and the theorem, we deduce

Corollary 3.3. The symmetric group $S_{n}$, for $n \geq 16$, is not the fundamental group of any closed orientable 3-manifold.

For $n=16$ this is the conclusion of Theorem 2.4, while for $n>16$ we use the observation that if $\pi \in \mathcal{G}^{3}$, then so is any finite index subgroup of $\pi$. 
It has been known since the work of Milnor Mil57 that a symmetric group cannot act freely on a homology sphere, and hence could not be the fundamental group of a 3-manifold, so that the corollary is certainly not new. Proofs and generalizations (along the lines of Theorem [2.5) of Milnor's result were given by R. Lee [Lee73] and J. Davis [Dav83] using the concept of the semicharacteristic $\chi_{\frac{1}{2}}$. In this vein, it seems quite suggestive that for a 3 -manifold, we have

$$
S(M)=\chi_{\frac{1}{2}}(M ; \mathbf{Q})-\chi_{\frac{1}{2}}\left(M ; \mathbf{Z}_{2}\right) .
$$

However, I do not see how to deduce Theorems 2.4 or 2.5 using more traditional methods of surgery theory.

Finally, the reader may wonder whether the invariant $\Delta \mathrm{S}$ can be used to decide the membership in $\mathcal{G}^{3}$ of those finite groups which remain for the moment out the reach of surgery theory. According to known theorems (see again DM85]), the key case to decide is that of the 'generalized quaternion groups' $Q(8 a, b, c)$. Unfortunately, our methods do not shed any light on this question.

Theorem 3.4. The groups $Q(8 a, b, c)$ all satisfy condition CS.

The proof of the theorem is a fairly tedious examination of the subgroup structure of $Q(8 a, b, c)$, and is omitted.

Remark 3.5. Larry Taylor made the following general observation concerning the applicability of our methods to the question of deciding membership in $\mathcal{G}^{3}$. Namely, if all of the surgery-theoretic obstructions vanish for a group $G$, then it must satisfy condition CS. The vanishing of the obstructions implies that $G$ acts freely on a homology sphere, and then Theorem 2.5 implies that condition CS holds. It would be interesting to know the precise relationship of the classical obstructions to the one derived from the Chern-Simons invariant.

\section{ACKNOWLEDGEMENT}

I had helpful conversations with Jerry Levine and Doug Park, and would also like to thank Robert Guralnick for a helpful correspondence about almost-conjugate subgroups, and Larry Taylor for his interesting remarks.

\section{REFERENCES}

[APS75a] M.F. Atiyah, V.K. Patodi, and I.M. Singer, Spectral asymmetry and Riemannian geometry: I, Math. Proc. Camb. Phil. Soc. 77 (1975), 43-69. MR 53:1655a

[APS75b] M.F. Atiyah, V.K. Patodi, and I.M. Singer, Spectral asymmetry and Riemannian geometry: II, Math. Proc. Camb. Phil. Soc. 78 (1975), 405-432. MR 53:1655b

[Bér92] Pierre Bérard, Transplantation et isospectralité. I, Math. Ann. 292 (1992), 547-559. MR 93a:58168

[CS74] S. Chern and J. Simons, Characteristic forms and geometric invariants, Annals of Math. 99 (1974), 48-69. MR 50:5811

[Dav83] James F. Davis, The surgery semicharacteristic, Proc. London Math. Soc. (3) 47 (1983), 411-428. MR 86a:57027

[DM85] J. F. Davis and R. J. Milgram, A survey of the spherical space form problem, Mathematical Reports, vol. 2, Harwood Academic Publishers, Chur, 1985. MR 87e:57001

[HS64] Jr. Hall, Marshall and James K. Senior, The groups of order $2^{n}(n \leq 6)$, The Macmillan Co., New York, 1964. MR 29:5889

[Lee73] R. Lee, Semicharacteristic classes, Topology 12 (1973), 183-199. MR 50:14809

[Mil57] John Milnor, Groups which act on $S^{n}$ without fixed points, Amer. J. Math. 79 (1957), 623-630. MR 19:761d 
[S+93] Martin Schönert et al., GAP - Groups, Algorithms, and Programming, Lehrstuhl D für Mathematik, Rheinisch Westfälische Technische Hochschule, Aachen, Germany, third ed., 1993.

[Sun85] T. Sunada, Riemannian coverings and isospectral manifolds, Annals of Math. 121 (1985), 169-186. MR 86h:58141

[Tho86] C.B. Thomas, Elliptic structures on 3-spheres, London Math. Soc. Lecture Note Ser., vol. 104, Cambridge Univ. Press, Cambridge, 1986. MR 87m:57015

Department of Mathematics, Brandeis University, Waltham, Massachusetts 02454

E-mail address: ruberman@brandeis.edu 